The International Conference on

Instrumentation for High Energy Physics

Dubna, USSR, Sept. 8-12, 1970
UCRL-20042

Preprint

CONA-700902-6

\title{
SEMICONDUCTOR DETECTORS IN
}

HIGH ENERGY PHYSICS

Clyde E. Wiegand

September 1970

AEC Contract No. W - 7405-eng -48
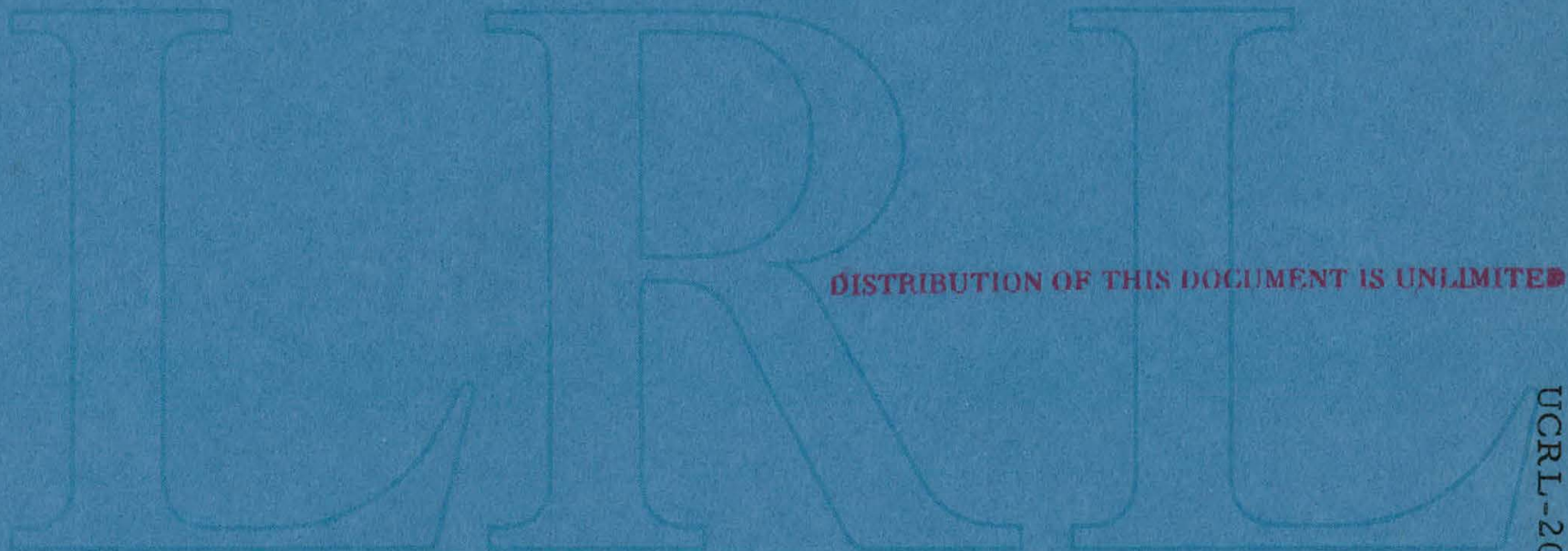

LAWRENCE RADIATION LABORATORY ఏ UNIVERSITY of CALIFORNIA BERKELEY 


\section{DISCLAIMER}

This report was prepared as an account of work sponsored by an agency of the United States Government. Neither the United States Government nor any agency Thereof, nor any of their employees, makes any warranty, express or implied, or assumes any legal liability or responsibility for the accuracy, completeness, or usefulness of any information, apparatus, product, or process disclosed, or represents that its use would not infringe privately owned rights. Reference herein to any specific commercial product, process, or service by trade name, trademark, manufacturer, or otherwise does not necessarily constitute or imply its endorsement, recommendation, or favoring by the United States Government or any agency thereof. The views and opinions of authors expressed herein do not necessarily state or reflect those of the United States Government or any agency thereof. 


\section{DISCLAIMER}

Portions of this document may be illegible in electronic image products. Images are produced from the best available original document. 


\author{
Clyde E. Wiegand
}

\author{
Lawrence Radiation Laboratory \\ University of California \\ Berkeley, California 94720
}

September 1970

Semiconductor detectors have been applied to the study of protonproton scattering at the highest energy now available in controlled beams, the Serpukhov accelerator. The experiment was described in the first session of this Conference by V. A. Nikitin.

Semiconductor detectors are being used at high energy accelerators in the study of kaonic and hyperonic atoms. They are also employed to identify isotopes of light elements that are ejected from heavy elements bombarded by high energy protons. Experiments have been made to study elastic scattering by placing solid-state detectors directly in a beam of high energy particles.

Semiconductor detectors have also been used to control the operation of a bubble chamber in an effort to increase the number of desired events per picture.

This paper should acquaint the reader with the present state of the development of semiconductors detectors and how they are being used in experiments at high energy accelerators.

Semiconductors detectors are used principally for the measurement of the energies of $x$ rays and $\gamma$ rays. Their main characteristics are excellent energy resolution together with high efficiency. For example: consider $x$ rays in the energy region of $100 \mathrm{keV}$ : a good germanium detector has an energy resolution of $1.4 \mathrm{keV}$ full width at half maximum (FWHM). It presents an area of about $10 \mathrm{~cm}^{2}$ to the incoming radiation, and has an intrinsic efficiency of 0.4. ("Intrinsic" efficiency implies that the radiation falls approximately perpendicularly on the face of the crystal, so that the solid angle subtended need not be considered.)

Semiconductor detectors are basically single crystals of germanium or silicon. The active volume of the crystal, in which the radiation is to be detected, must be of high electrical resistance. Although the detectors are 
made from materials of higher purity than is ordinarily used in other solidstate devices, such as transistors, it has not been possible to produce ultrapure crystals large enough for practical detectors. However, while investigating a basic problem in diffusion rates, Pell [1] found that ions could be made to drift in the electric field of $p-n$ junctions. He found that a high-resistance semiconductor region could be produced between $n$ - and p-type layers. Researchers in radiation detectors recognized that ion drifting should permit production of larger volumes of high-resistance (intrinsic) germanium; fairly large semiconductor crystals can now be made.

The manufacture begins with germanium (or silicon) that contains an excessive number of acceptor atoms. Lithium is then drifted through the crystal under the force of an electrific field until the number of donors balances the number of acceptors. This process of compensation is called lithium drifting and hence the designation lithium-drifted germanium, Ge( Li). Many reports have been published on the techniques of producing high-resistance crystals by lithium sompensation [2]:

The drifting process is so slow that it takes weeks to compensate a volume. of about $10 \mathrm{~cm}^{3}$; unfortunately, only a small fraction of the crystals whose resistivity and structure appear to be satisfactory make good radiation detectors. Electrons and holes are trapped by various pitfalls as they move through a crystal; mechanical strain induced by thermal stress appears to be the dominant factor in preventing good ion mobility. Not all the characteristics that a crystal must possess to make an excellent radiation detector are understood. However, in the last year or two significant progress has been made in recognizing, at an early stage of manufacture, germanium crystals that will make excellent detectors [3].

This paper is concerned mainly with the application of germanium and silicon detectors of the lithium-drifted variety that are used for the energy ...... 1 
measurement of $x$ rays and $\gamma$ rays in the region of $30 \mathrm{keV}$ to $3 \mathrm{MeV}$.

The principle of operation is as follows (refer to Fig. 1): Radiation enters the active volume, where it undergoes Compton scattering, ejects photoelectrons, and--if the energy is high enough--produces electron-positron pairs. The kinetic energy of the electrons and positrons is absorbed by the germanium, and results in ionization of some of the germanium atoms along the tracks of the electrons. The ions consist of electrons knocked free from the germanium atoms and the remaining positively charged atoms that have electron vacancies (holes). An average of $2.97 \mathrm{eV}$ of kinetic energy is absorbed for each hole-electron pair produced in $\mathrm{Ge}(\mathrm{Li})$, and an average of $3.8 \mathrm{eV}$ in $\mathrm{Si}(\mathrm{Li})$. The electrons drift to the positively charged surface of the crystal and the holes drift to the negatively charged surface. The amount of charge is measured by a charge-sensitive preamplifier. The pulse output of the amplifier is proportional to the charge and inversely proportional to the effective capacitance of the crystal plus the amplifier input circuit.

Several comprehensive papers on the theory and operation of semiconductor detectors and their associated electronic apparatus have been published. A review by Tavendale, the papers by Goulding, Landis, and Pehl, and the book edited by Bertolini and Coche are recommended [4].

Energy resolution is of supreme importance in $x$-ray and $\gamma$-ray spectroscopy. Let us review some recent accomplishments. In the low-energy region (a few keV), Landis, Goulding, and Jaklevic [5], using a Si(Li) detector, $0.5 \mathrm{~cm}$ in diameter and $0.3 \mathrm{~cm}$ thick, achieved a line width of $105 \mathrm{eV}$ at an energy of $1740 \mathrm{eV}$. Figure 2 shows the spectrum of several x-ray lines and the electronic resolution, which was $91 \mathrm{eV}$ with the detector connected to the preamplifier. This low value of electronic noise was achieved by employing a pulsed optoelectronic feedback preamplifier in which light was used to couple the electronic feedback circuit to the input point of the preamplifier. 
For the energy region of $1 \mathrm{MeV}$, Fig. 3 illustrales the peak-to-Compton ratio for a $15-\mathrm{cm}^{3} \mathrm{Ge}(\mathrm{Li})$ detector [6]. Figure 4 shows the energy resolution of a $0.4 \times 0.4 \times 0.4-\mathrm{cm}^{3} \mathrm{Ge}(\mathrm{Li})$ detector employing optoelectronic feedback [7]. We see a resolution of almost 1 part in 1000 at $1.4 \mathrm{MeV}$.

Some characteristics of semiconductor detector systems should be pointed out. In Fig. 3, we note that in order for an $x-r$ ay photon to be measured all its energy must be contained within the sensitive region of the detector crystal. If any of the energy leaks out, the collected charge is reduced and the pulse height is lowered. The background under the peaks comes mainly from radiation leakage. The ultimate resolution of the system is determined by the noise of the preamplifier, the fluctuation in leakage currents, and the statistical fluctuation in the number of electron-hole pairs produced. Even though a definite amount of energy be dissipated in the detector, the number of holeelectron pairs produced is not exact. The reason is that not all the kinetic energy is used to make electron-hole pairs. Some of the energy gnes into light, heat, and sound. It is found that fluctuations in detectors are described by $\Delta E(F W H M)=2.36 \epsilon(F\langle N\rangle)^{\frac{1}{2}}=2.36(F E \epsilon)^{\frac{1}{2}}$, where $\epsilon=$ the average energy for the production of a hole-electron pair, $F$ is the Fano factor, and $\langle N\rangle$ is the average number of hole-electron pairs. Ge( Li) detectors have been made with $F$ as low as $0.08[8]$.

Another characteristic of the detectors of interest to physicists is the time resolution of semiconductor systems. The mobility of the charges is such that in a typical detector $1 \mathrm{~cm}$ thick, the time for collection is about $50 \mathrm{nsec}$. The resolution curve is much narrower than the total time of collection of the charges. For a Ge(Li) detector in planar configuration with a sensitive depth of $1 \mathrm{~cm}$ and an area of $8 \mathrm{~cm}^{2}$, the time resolution achieved was, for all pulses greater than $20 \mathrm{keV}$ in energy, $5.6 \mathrm{nsec} F W H M$ and $12 \mathrm{nsec} F \mathrm{~W}(0.1) \mathrm{M}$. A Ge( Li) detector in coaxial configuration showed a resolution of $9.8 \mathrm{nsec}$ FWHM and 
23 nsec FW(0.1)M. The above data were obtained by Jaklevic, Bernthal, Radeloff, and Landis [9], who employed a leading-edge discriminator to extract the timing information. Separate circuitry was used to comepnsate for the energy dependence of the timing signal. However, the amount of time necessary to make an accurate energy measurement is about $1 \mu \mathrm{sec}$. The 1 - $\mu$ sec interval is necessary to assure that each pulse has the optimum shape for the highest signal-to-noise ratio.

Let us look at an experiment that uses particles that can be produced only at high-energy accelerators [10]. The experiments at the Berkeley Bevatron are, of course, the ones with which I am most familiar, and I will describe one of them as an example. Figure 5 is intended to show the arrangement of the experiment to study some nuclear properties by measurement of the $x$ rays emitted by hydrogen-like atoms that consist of a kaon orbiting around a nucleus.

About 30 kaons were stopped per second in the target between the detectors. Eachkaon formed a hydrogen-like system that existed for only about $10^{-12}$ sec. During the se brief intervals the kaons cascaded toward their host nucleus and emitted $x$ rays analogous to the $K, L, M, \cdots, x$ rays emitted by an ordinary hydrogen atom, except that here the energies were about 1000 times as great. We used three semiconductor detectors to cover the energy range from $15 \mathrm{keV}$ to $550 \mathrm{keV}$. A lithium-drifted silicon detector $3 \mathrm{~mm}$ thick was used to measure the energies of $x$ rays up to $60 \mathrm{keV}$; the $1.3-\mathrm{cm}-$ thick $\mathrm{Ge}(\mathrm{Li})$ in planar geometry was designed to work best over the range $60 \mathrm{keV}$ to about $300 \mathrm{keV}$; a $\mathrm{Ge}(\mathrm{Li})$ detector in coaxial configuration was employed to measure more efficiently the energies above $300 \mathrm{keV}$. It is difficult to make one detector cover the entire range of energies.

The telescope of scintillation counters and a Cerenkov counter registered the kaons stopped in the target. Each signal of a stopped kaon was put in coincidence with signals from the detectors. The coincidence output signal then 
opened a linear gate between the amplifier and the data-storage system. In addition to the coincidence circuits, the pulses bearing time information were processed by a Pile-Up Rejector. The pile-up rejector certified that the amplifier was free of interference pulses that might have occurred before or after the pulse whose height was to be measured. After passing the linear gate the pulses from each detector were sent to a multiplexer where they were lengthened in time and held until accepted by one analog-to-digital converter common to the 4096 storage channels. The pulse height of each detector signal was measured and stored in a magnetic core memory. Between accelerator bursts the accumulated energy measurements along with their serial numbers were transferred to magnetic tape. Finally the tapes were taken to a computer for processing into $x$-ray spectra, of which Fig. 6 is an example.

Hyperons are produced when negative kaons are captured by nucleons at the surfaces of nuclei. Some of the hyperons are $\Sigma^{-}$particles that are ejected from the nucleus in which they are produced. Most of the $\Sigma^{-}$particles thus produced stay within the target and can be captured by target nuclei into $\Sigma^{-}$hyperonic atoms. These alsoare hydrogen-like systems and emit $x$ rays as the hyperons cascade toward their host nuclei. There is one important difference between the kaonic atoms and the hyperonic atoms: kaons have spin zero. whereas $\Sigma^{-}$particles have spin $1 / 2$. This means that the $x$-ray spectral lines will be split into doublets. The amount of splitting depends upon the magnetic moment of $\Sigma^{-}$, which has not been measured. It would be important to high energy particle physics to measure the magnetic moment, its value is predicted by theory. An $\mathbf{x}$-ray spectral line of $\Sigma^{-}$-hyperonic atoms was first observed among the kaonic $\mathbf{x}$-ray lines at Berkeley, and later another was seen in a CERN kaonic $x$-ray spectrum [10]. These first hyperonic atom $x$ rays were observed in light elements, in which the splitting is too small for observation. It will be necessary to generate intense lines in a heavy element before 
the splitting can be seen. There is at least one other kind of atom that can be studied: antiprotons when brought to rest in matter should make antiprotonic atoms. The spin of the antiprotons should produce a splitting of the lines that would be observable with semiconductor detectors now in use. At this writing no antiprotonic $x$-ray lines have been reported.

The magnetic moment of antiprotons is expected to be opposite in algebraic sign and of the same magnitude as the magnetic moment of protons. The magnitude of the magnetic moment of $\Sigma^{-}$is expected to be different from the magnetic moment of $\Sigma^{+}$.

Semiconductor detectors have been used at Berkeley by Poskanzer, Butler, Hyde, and Cerny to discover new isotopes of the light elements [11]. An external beam of $5.5-\mathrm{GeV}$ protons from the Bevatron bombarded a $27-\mathrm{mg} / \mathrm{cm}^{2}$ uranium foil and ejected isotopes such as ${ }^{14} \mathrm{~B},{ }^{15} \mathrm{~B}$, and ${ }^{17} \mathrm{C}$. A telescope of thin phosphorus-diffused silicon counters was used to identify the ejected particles. The identification was accomplished by measurement of $-\mathrm{dE} / \mathrm{dx}$, total energy, and velocity of the particles. The velocity measurements were made by time of flight over a distance of $20 \mathrm{~cm}$. The time resolution varied from $0.3 \mathrm{nsec}$ for ${ }^{15} \mathrm{~N}$ up to $0.5 \mathrm{nsec}$ for ${ }^{7} \mathrm{Be}$. Figure 7 shows a spectrum obtained by the Berkeley group.

An experiment to check the feasibility of placing a semiconductor detector directly in a high energy beam was made at Berkeley by Lander, Mehlhop, Lubatti, and Schnurmacher [12]. In this experiment it was necessary to know whether or not high energy protons made elastic or inelastic collisions. A lithiumdrifted silicon detector was placed in a collimated beam of protons and functioned as a scattering target. Ejected particles were detected in coincidence with the signals from the semiconductor detector. If the collision were elastic the energy deposited in the detector would be essentially unique and consist of the sum of itheirecoil energy of a silicon nucleus and the energy lost by the high 
energy proton. If the collision were inelastic the energy deposited would depend upon the nature of the collision.

Recently, with a similar idea but a more refined and accurate experiment, Bellini, di Corato, Manfredi and Vegni [13] demonstrated pure coherent pion production by $15 \mathrm{GeV} / \mathrm{c}$ negative pions incident on Si nuclei. They employed a stack of $5 \mathrm{Si}$ detectors as the target in a pion beam at CERN. Each of the 5 detectors was connected individually to circuits that digitized their output pulses and recorded the pulse height information on magnetic tape. In addition a kinematical fit to the production of 3 charged pions was required of spark chambers that recorded the outgoing particles. The data were analyzed by making the additional requirements that une only of the Si detectors showed a pulse and that less than $1.5 \mathrm{MeV}$ was deposited by the recoil nucleus. The corresponding four-momentum transfer to the nucleus was $\sim 0.08(\mathrm{GeV} / \mathrm{c})^{2}$. The solid lines of Fig. 8 show the distribution of events. For comparison the distribution is shown for the same data, but without selection by the semiconductor detectors. The energy resolution of the Si detectors was $60 \mathrm{keV}$ and in all events the average energies of the incident and outgoing fast particles were subtracted. It is evident that this method permits the study of the characteristics of pure coherent production.

Another use of semiconductor detectors in high energy physics is reported in a paper submitted to this Conference [14]. At the Stanford Linear Accelerator Center, the 82 -inch bubble chamber was tested under the control of a semiconductor detector and scintillation counters. A beam of pions was defined by a collimator and scintillation counters in front of the bubble chamber. A semi. conductor detector was placed inside the liquid hydrogen contained at the rear wall of the chamber. The idea was to allow a single particle to enter the 
chamber and to take a picture only if a scattering occurred; a particle that went straight through the chamber would strike the solid-state detector and produce a veto signal. It is planned to install an array of semiconductor detectors in the bubble chamber in an effort to delineate the tracks. A programmed spacing of the useful tracks would simplify the analysis, especially when automatic scanning devices are employed.

Ordinarily the short duration of the beam bursts of linear accelerators makes difficult the operation of semiconductor detector systems. Afanas'ev, Bochagov, Semenchuk and Solyakin of the Ioffe Physical-Technical Institute [15] have devised a method of background compensation that allows the investi -: gation of photonuclear reactions with cross sections of the order of $10^{-27} \mathrm{~cm}^{2}$. The basic idea of the scheme is shown in Fig. 9. A $\gamma$-ray beam from an electron accelerator was incident on a target from which it was desired to measure the energy of photon-induced recoils. Two detectors were placed near to each other so that they both received the same electron background. One detector was the "working" detector and the other the "compensating" detector. The bias of the detectors was so arranged that pulses of opposite sign were fed simultaneously to the input of one charge sensitive preamplifier. The characteristics of the detectors were made as nearly equal as possible and the electronic circuits were tuned for best cancellation of background. In summary, we see that semiconductor detectors are being used in high energy physics and especially in a rather new kind of experiment that brings together high energy physics and nuclear structure as well as the study of elementary particle properties. The proceeding of the Third International Conference of High Energy Physics and Nuclear Structure [16] describes more of this lype of research. 
References

1. E. M. Pell, J. Appl. Phys. 31, 291 (1960).

2. Lithium-Drifted Germanium Detectors, Proceedings of a Panel (International, Atomic Energy Agency, Vienna, June 6-10, 1966).

3. W. L. Ilangen, R. H. Pehl, E. J. Rivet, and F. S. Goulding, Nucl. Instr. Methods 80, 181 (1970).

4. A. J. Tavendale, Ann. Rev. Nucl. Sci. 17, 73 (1967); Fred S. Goulding, Nucl. Instr. Methods $\underline{43}, 1(1966) ;$ F. S. Goulding, D. A. Landis, and R. H. Pehl, in Semiconductor Nuclear-Particle Detectors and Circuits (National Academy of Sciences, Washington, 1969) p. 455; G. Bertolini and A. Coche, Eds., Semiconductor Detectors (North-Holland Publishing Company, Amsterdam, 1968).

5. D. A. Landis, F. S. Goulding, and J. M. Jaklevic (Lawrence Radiation Laboratory Report, UCRL-19796, June 1970), submitted to Nucl. Instr. Methods; K. Kandiah and A. Sterling in Semiconductor Nuclear-Particle

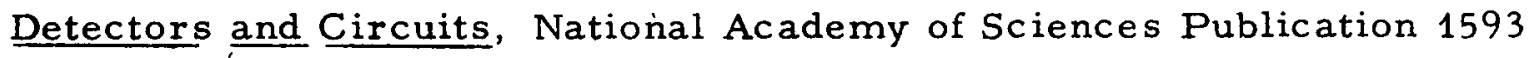
p. 495 (1969); V. Radeka, IEEE Trans. Nucl. Sci. NS-17 [3], 433 (1970).

6. F. S. Goulding, D. A. Landis, and R. H. Pehl, in Semiconductor Nuclear Particle Detectors and Circuits (National Academy of Sciences, Washington, 1969).

7. F. S. Goulding, J. T. Walton, and R. H. Pehl, IEEE Trans. Nucl. Sci. NS $-17[1], 218(1970)$.

8. Richard H. Pehl and Fred S. Goulding, Nucl. Instr. Methods $\underline{81}, 329$ (1970); F. J. Walter in Space Semiconductor Nuclear-Particle Detectors and Circuits, National Academy of Sciences Publication 1593, p. 63 (1969). 
9. J. M. Jaklevic, F. M. Bernthal, J. O. Radeloff, and D. A. Landis, Nucl. Instr. Methods 69, 109 (1969); F. S. Goulding, D. A. Landis, J. Cerny; and R. H. Pehl, Nucl. Instr. Methods 31, 1 (1964).

10. Clyde E. Wiegand and Dick A. Mack, Phys. Rev. Letters 18, 685 (1967); Clyde E. Wiegand, Phys. Rev. Letters 22, 1235 (1969); G. Backenstoss, A. Bamberger, J. Egger, W. D. Hamilton, H. Koch, U. Lynen, H. G. Ritter, and H. Schmitt, Phys. Letters B, 32 399 (1970).

11. A. M. Poskanzer, G. W. Butler, E. K. Hyde, J. Cerny, D. A. Landis, and F. S. Goulding, Phys. Letters B27, 414 (1968).

12. Richard L. Lander, W. A. W. Mehlhop, H. J. Lubatti, and Gerald L. Schnurmacher, Nucl. Instr. Methods 42, 261 (1966).

13. G. Bellini, M. diCorato, P. F. Manfredi, and G. Vegni, submitted to International Conference on Instrument for High Energy Physics (Dubna, U.S.S. R., September 1970).

14. D. H. Brick, D. Goloskie, I. A. Pless, E. Sartori, B. Wadsworth, R. A. Gearhart, J. J. Murray, and H. J. Lubatti, in Proceedings of International Conference on Instrumentation for High Energy Physics (Dubna, U.S.S. R., September 1970).

15. A. P. Komar, B. A. Bochagov, A. A. Kotov, Yu. N. Ranyuk, G. G. Semenchuk, G. E. Solyakin, and P. V. Sorokin, Yadernaya Fizica (Sov.) 10, $5 \underline{1}$ (1969).

16. Samuel Devons, Ed., Proceedings of the Third International Conference on High Energy Physics and Nuclear Structure (Plenum Press, New York, 1970). 


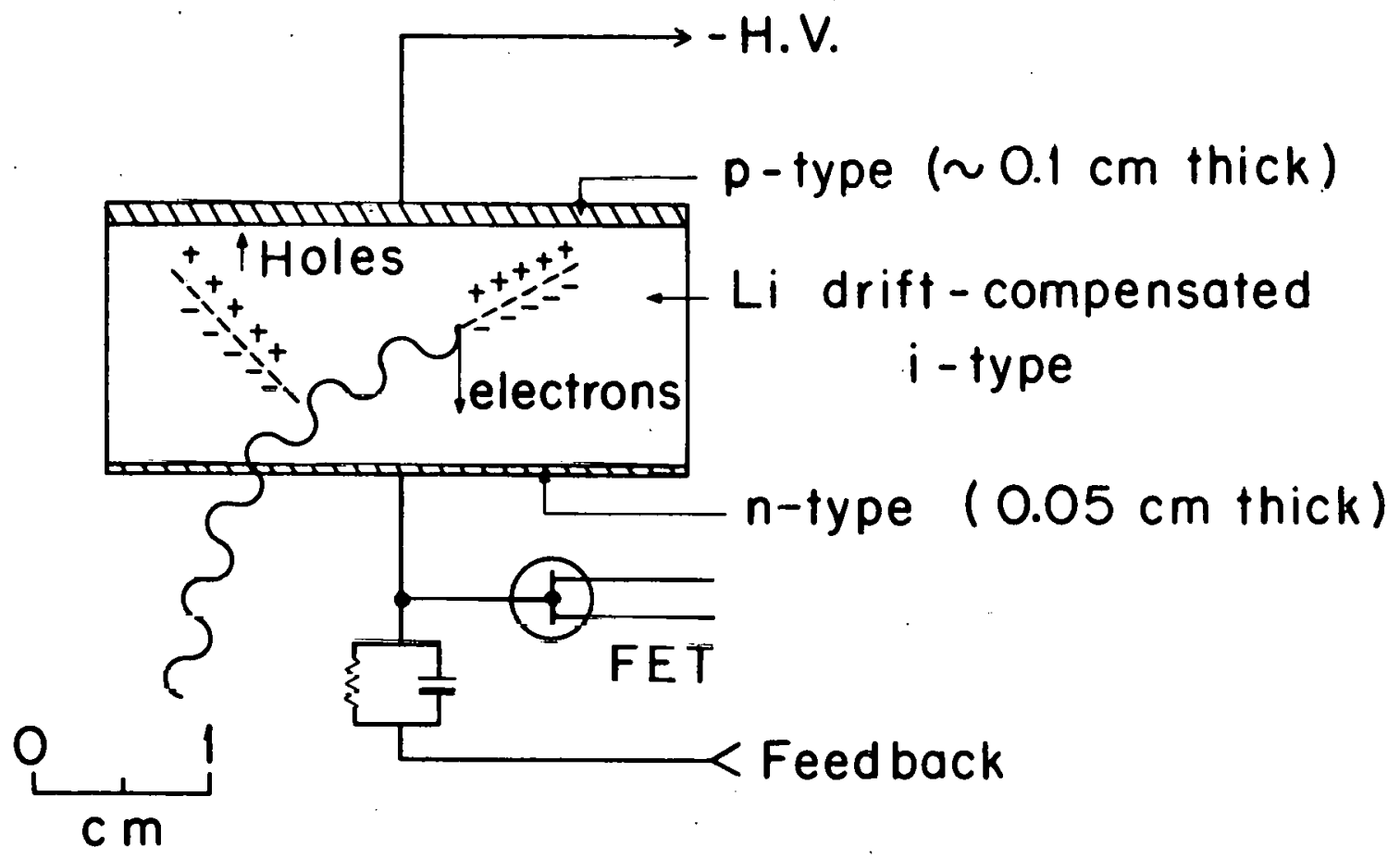

XBL708-3606

Fig. 1. Lithium-drifted germanium detector in planar configuration. 


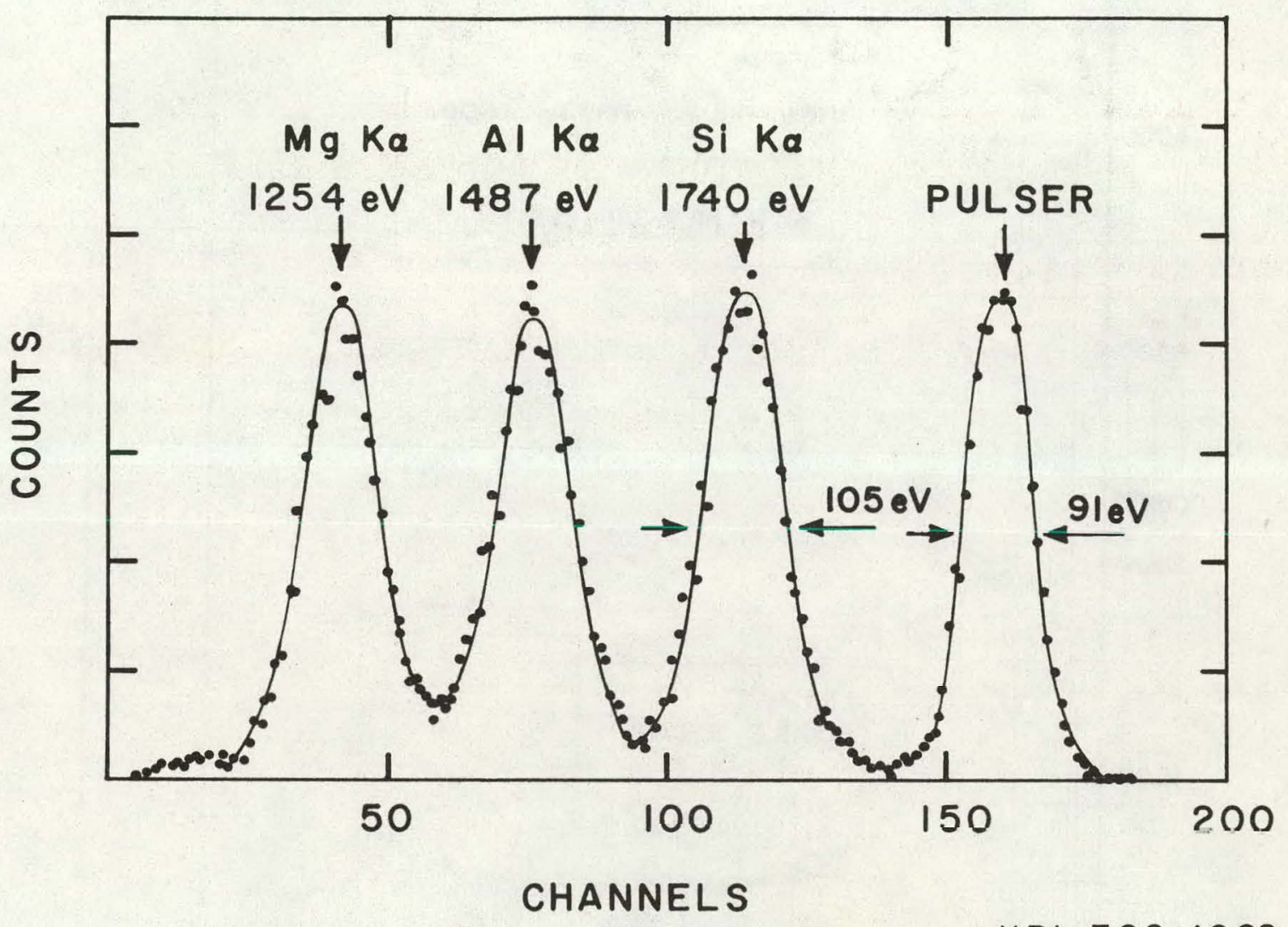

Fig. 2. Energy spectrum of $x$ rays in the $1.5-\mathrm{keV}$ region, taken by a $\mathrm{Si}(\mathrm{Li})$ detector. (Landis, Ref. 5.) 


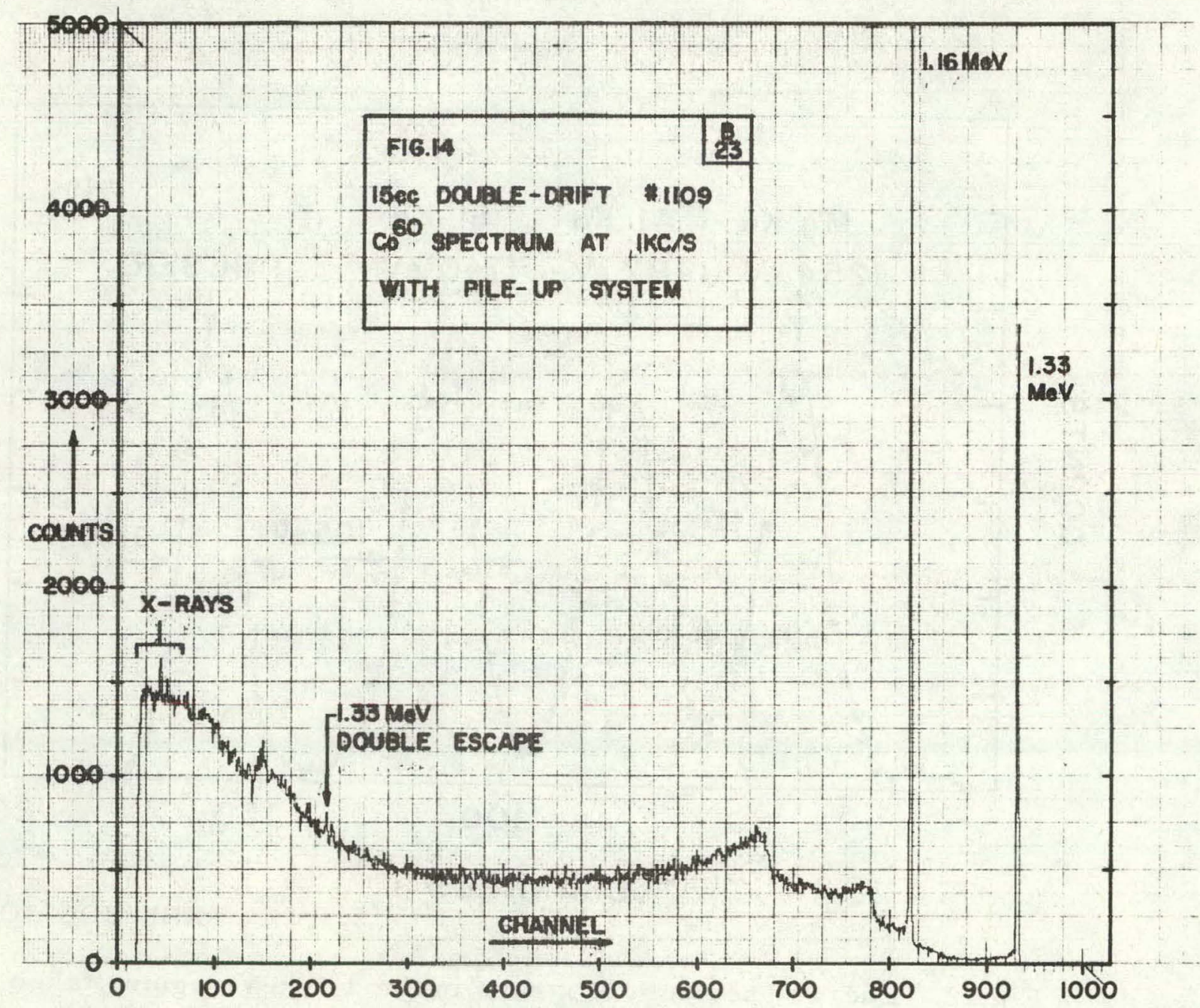

XBL $675-1481$

Fig. 3. Response to $\gamma$ rays from a ${ }^{60}$ Co source of a Ge( Li) detector in planar configuration. Goulding, Ref. 6.) 


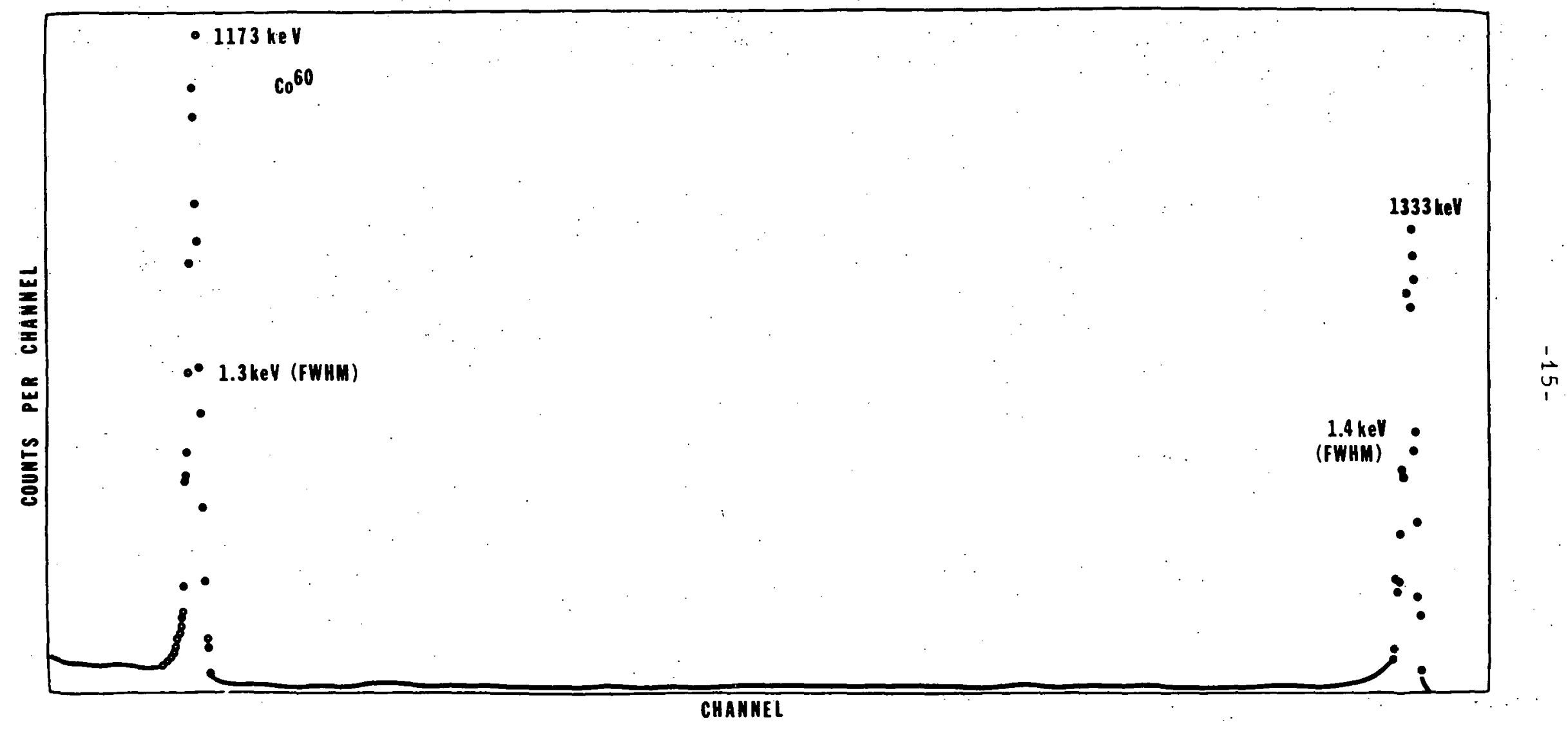

Fig. 4. Energy spectrum of ${ }^{60} \mathrm{Co} \gamma$ rays taken by a $\mathrm{Ge}(\mathrm{Li})$ detector

XBL $708-1695$ in planar configuration. (Goulding, Ref. 7.) 


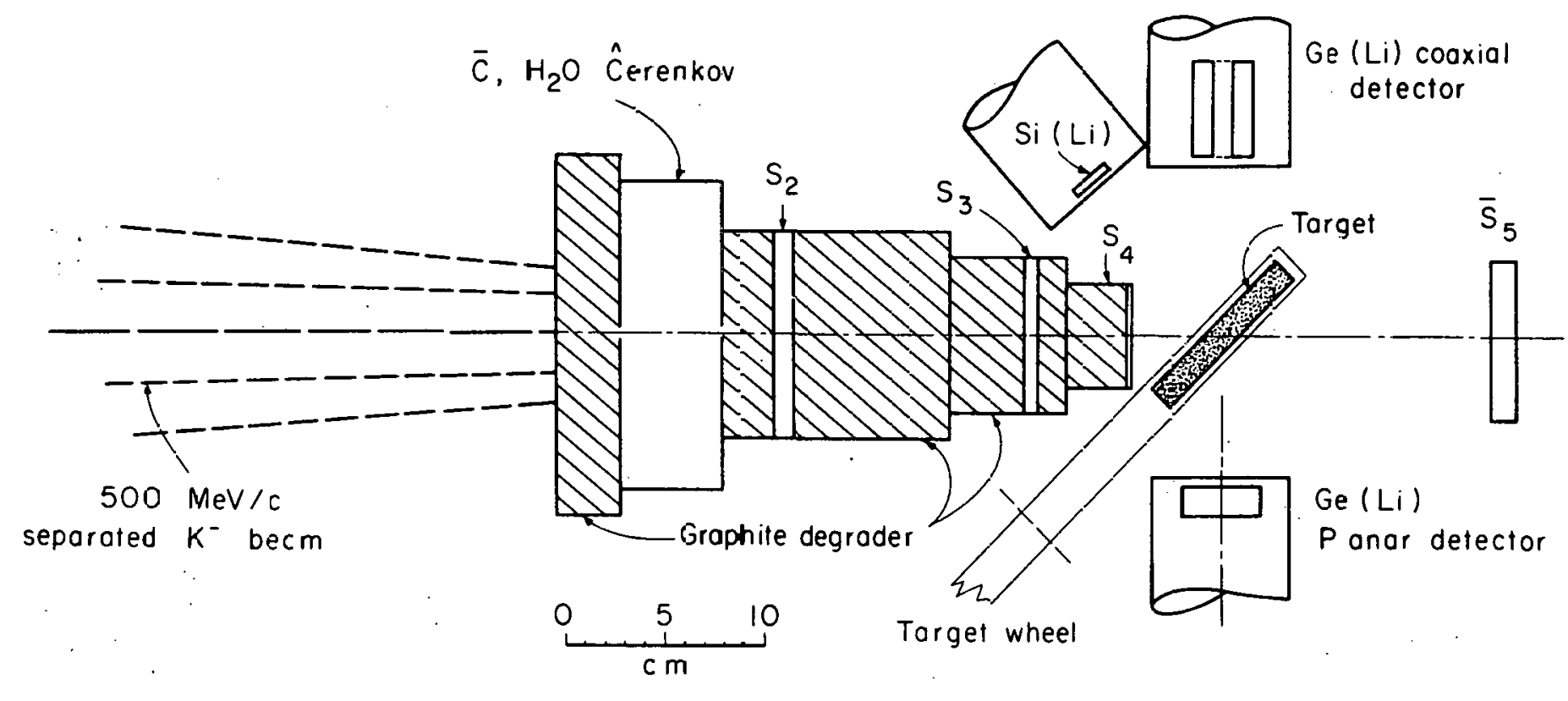

XBL $708-3573$

Fig. 5. Arrangement of, apparatus in a kaonic atom $\mathbf{x}$-ray experiment. 


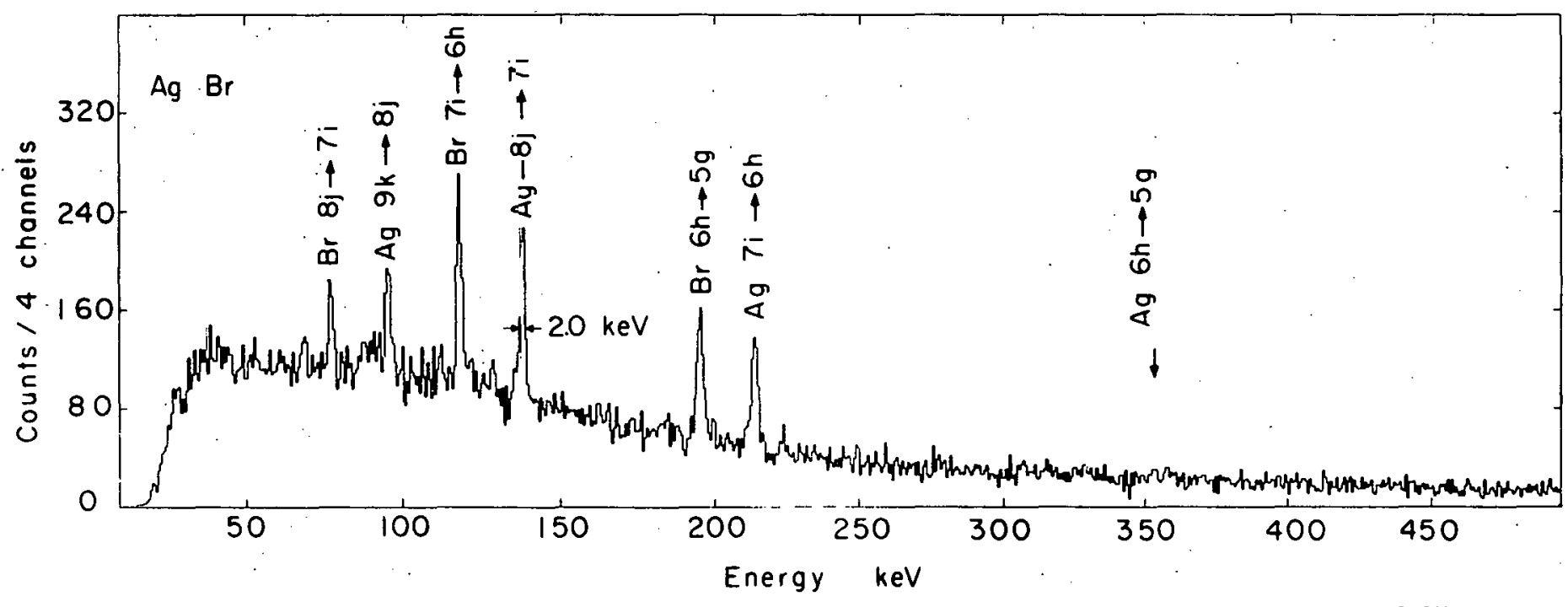

Fig. 6. Kaonic atom $x$-ray spectrum of AgBr. The transition in $\mathrm{Ag}$ from $\mathrm{n}=6$ to $\mathrm{n}=5$ is not observed. 


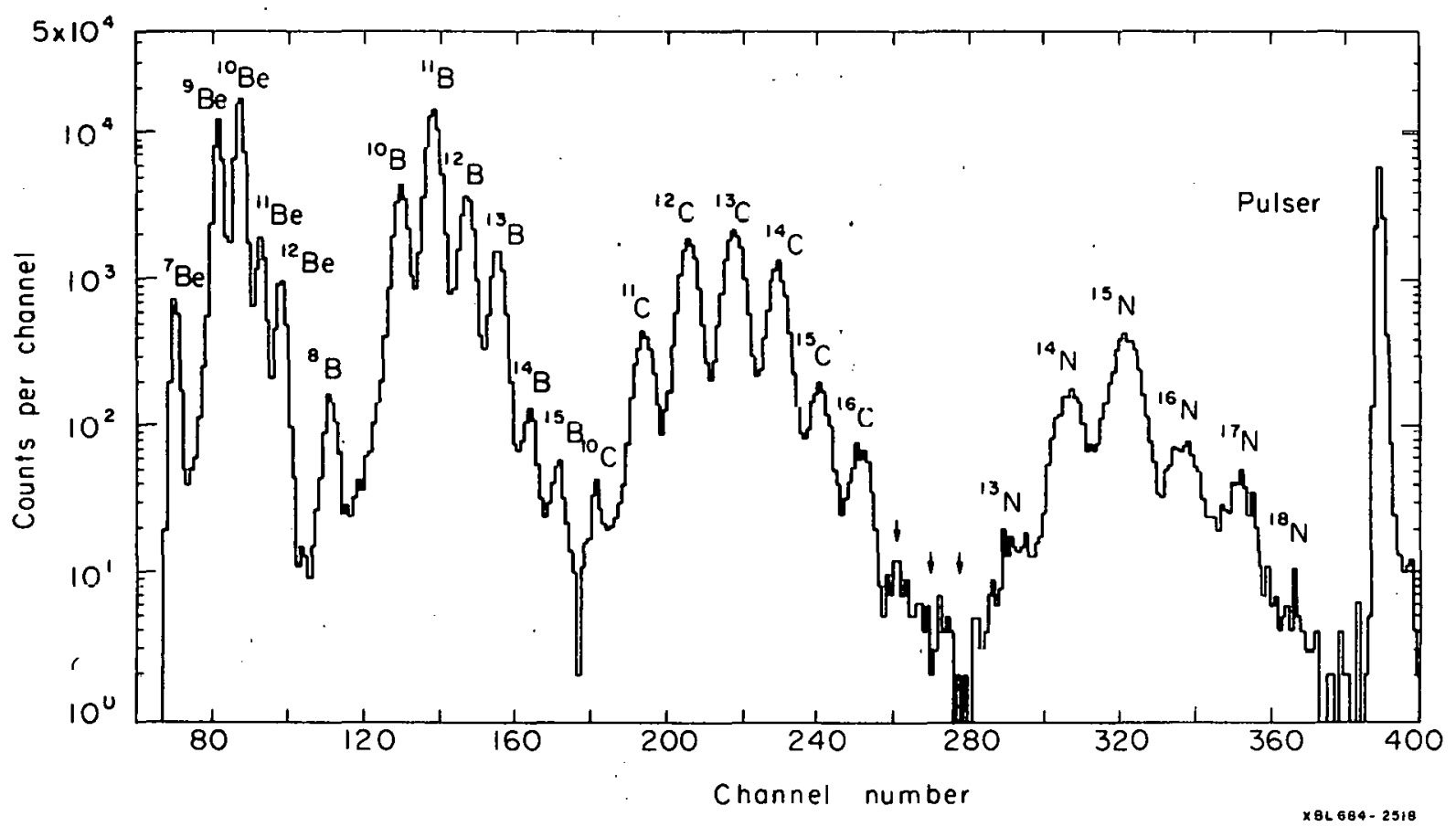

Fig. 7. Particle spectrum taken from energy-loss measurements in semiconductor detectors. (Poskanzer, Ref. 11.) 


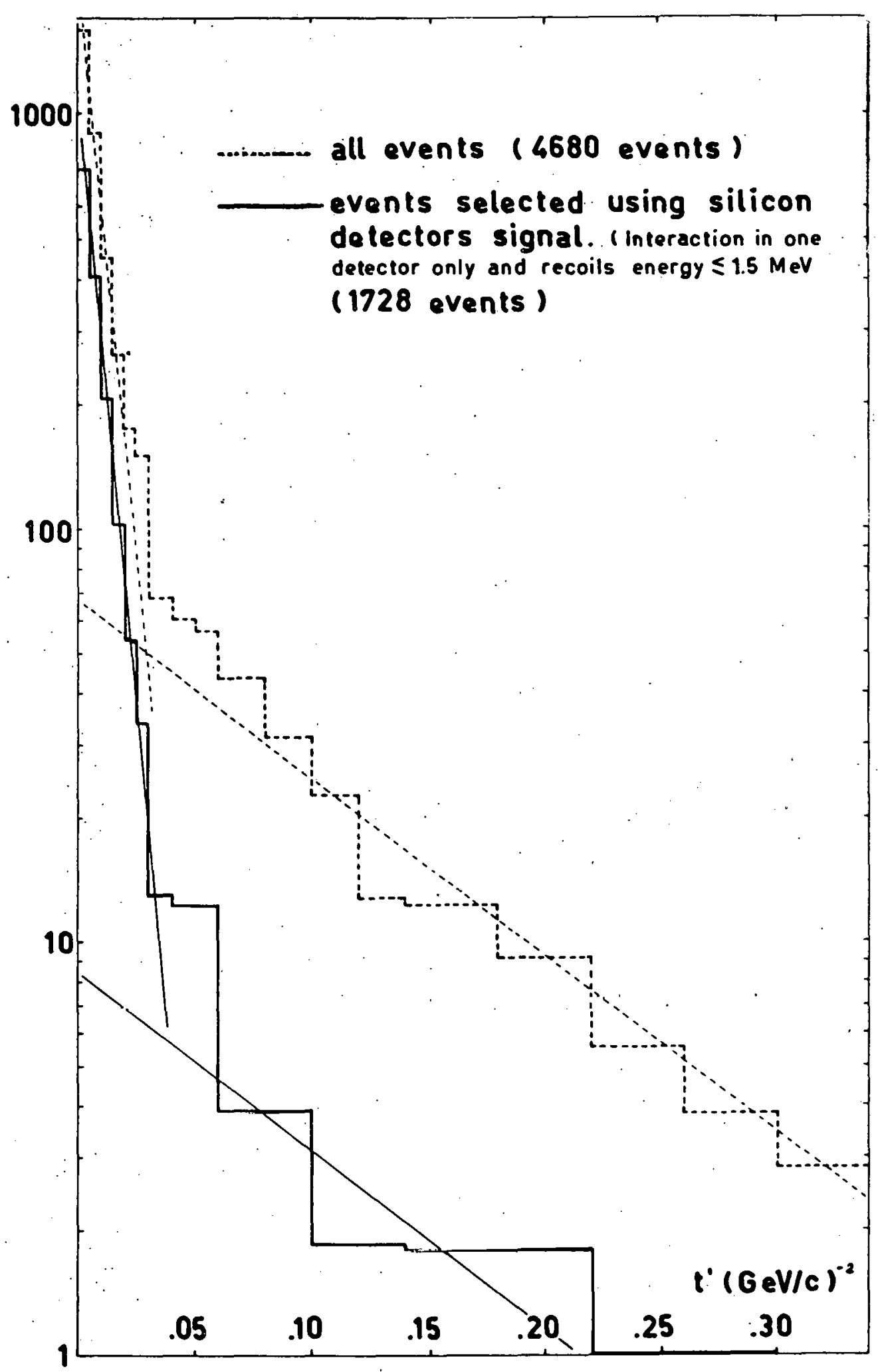

Fig. 8. Distribution of events in the coherent production of pions by a $15 \mathrm{GeV} / \mathrm{c}$ pion beam. The target was a Si semidetector. (Bellini, Ref. 13.) 


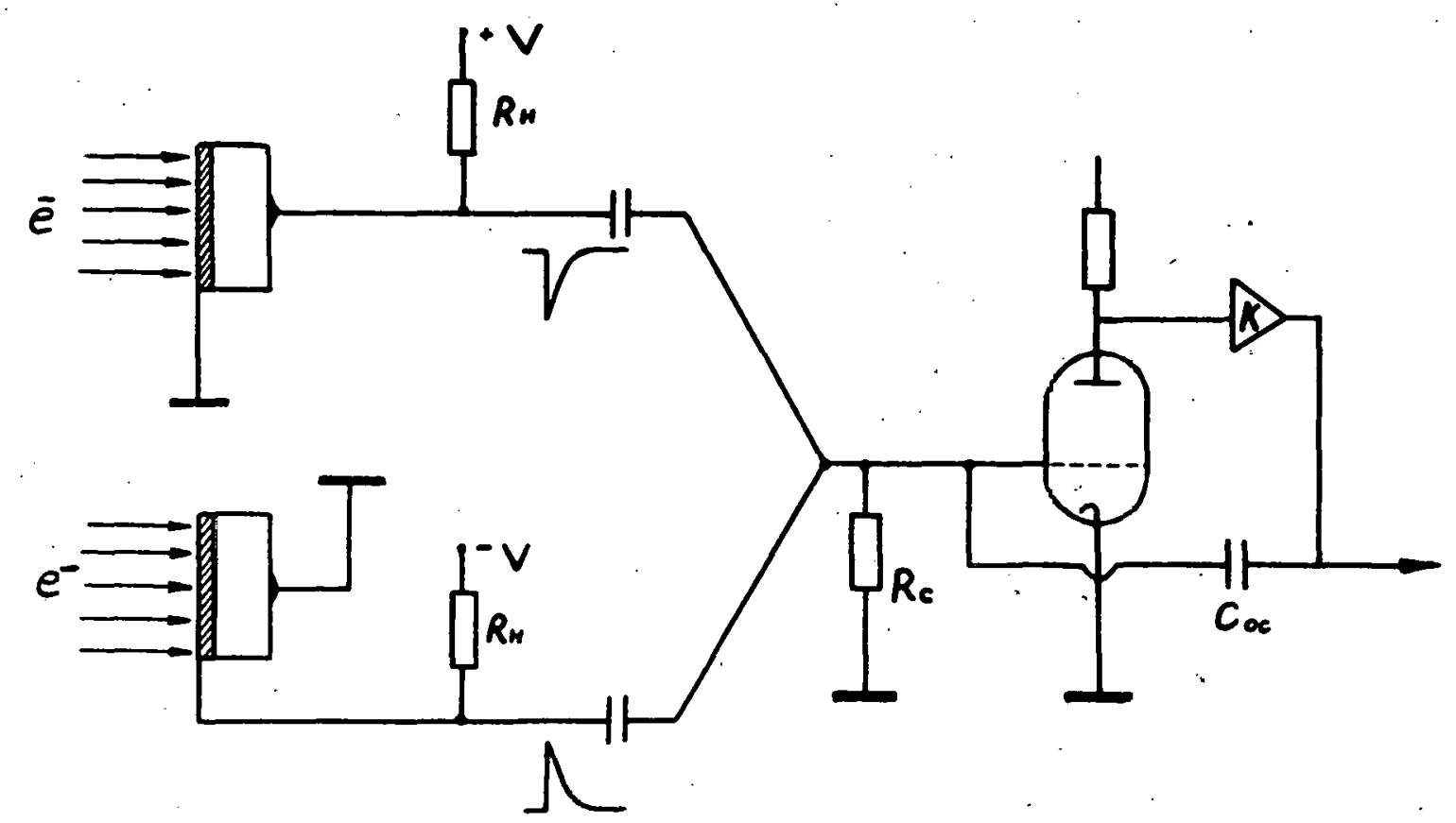

$\stackrel{1}{\sim}$

Fig. 9. Schematic comnection of detectors for background cancellation.

(Afanas'ev, Ref. $\mathbf{1}_{4 .}$ ) 
This report was prepared as an account of Government sponsored work. Neither the United States, nor the Commission, nor any person acting on behalf of the Commission:

A. Makes any warranty or representation, expressed or implied, with respect to the accuracy, completeness, or usefulness of the information contained in this report, or that the use of any information, apparatus, method, or process disclosed in this report may not infringe privately owned rights; or

B. Assumes any liabilities with respect to the use of, or for damages resulting from the use of any information, apparatus, method, or process disclosed in this report.

As used in the above, "person acting on behalf of the Commission" includes any employee or contractor of the Commission, or employee of such contractor, to the extent that such employee or contractor of the Commission, or employee of such contractor prepares, disseminates, or provides access to, any information pursuant to his employment or contract with the Commission, or his employment with such contractor. 
